\title{
Surface Etching of Polymeric Semiconductor Films Improves Environmental Stability of Transistors
}

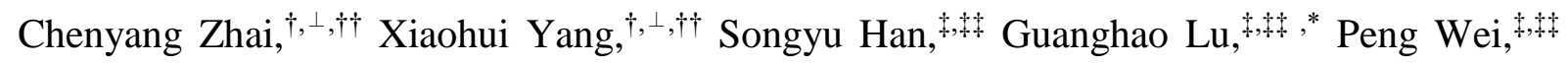
Andrei Chumakov, ${ }^{\S}$ Elisabeth Erbes,${ }^{\S}$ Qing Chen, ${ }^{\S}$ Simone Techert, ${ }^{\S}$ Stephan V Roth,,${ }^{\S}$ Peng Zhang, ", Laju Bu ${ }^{\dagger, \perp, \dagger, *}$

${ }^{\dagger}$ School of Chemistry, Xi'an Jiaotong University, Xi'an, Shaanxi 710049, China

${ }^{\ddagger}$ Frontier Institute of Science and Technology, Xi'an Jiaotong University, Xi'an, Shaanxi 710054, China

§Deutsches Elektronen-Synchrotron (DESY), Notkestrasse 85, Hamburg D-22607, Germany

"School of Material Science and Engineering, PCFM lab, Sun Yat-sen University, Guangzhou, Guangdong 510275, China

${ }^{\perp}$ MOE Key Laboratory for Nonequilibrium Synthesis and Modulation of Condensed Matter, Xi'an Jiaotong University, Xi'an, Shaanxi 710049, China

†Xi'an Key Laboratory of Sustainable Energy Material Chemistry, Xi'an Jiaotong University, Xi'an, Shaanxi 710049, China

\#State Key Laboratory of Electrical Insulation and Power Equipment, Xi'an Jiaotong University, Xi'an, Shaanxi 710049, China

${ }^{\#}$ KTH Royal Institute of Technology, Department of Fibre and Polymer Technology, Teknikringen 56-58, SE-100 44 Stockholm, Sweden

*E-mail: guanghao.lu@mail.xjtu.edu.cn; zhangpeng3@mail.sysu.edu.cn; Laju2014@ mail.xjtu.edu.cn. 
(a)

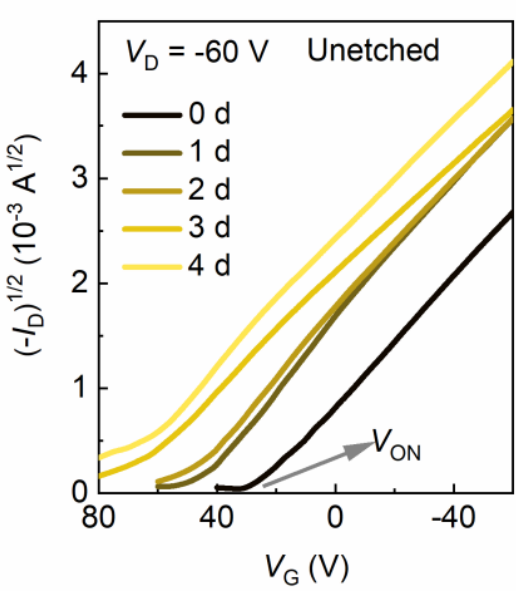

(b)

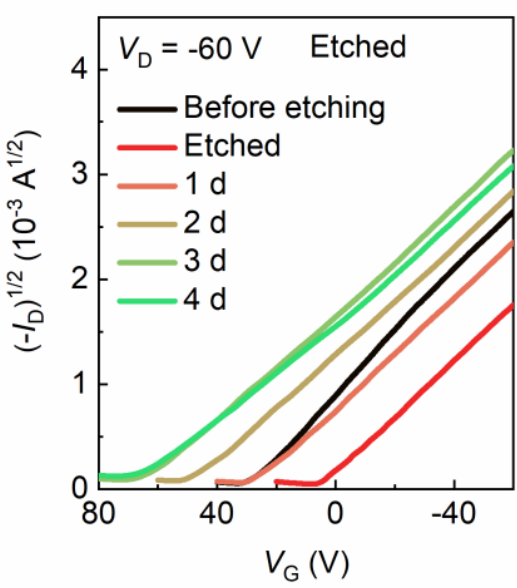

Figure S1. In situ evolution of transfer curves $\left(\left(-I_{\mathrm{D}}\right)^{1 / 2}-V_{\mathrm{G}}\right)$ for (a) unetched and (b) etched $\mathrm{P} 3 \mathrm{HT}$ transistors during exposure to air. The arrow shows the $V_{\mathrm{ON}}$. 
(a)

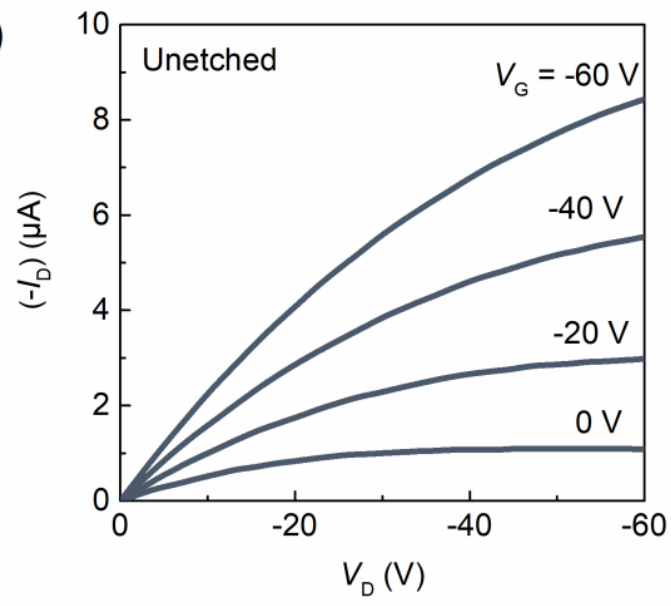

(b)

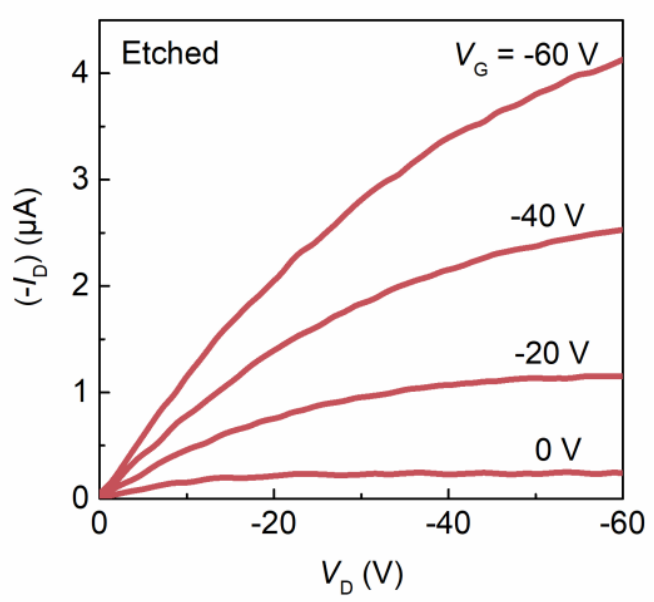

Figure S2. Output curves of (a) unetched and (b) etched P3HT transistors. The transfer curves are shown in Figure 2. 

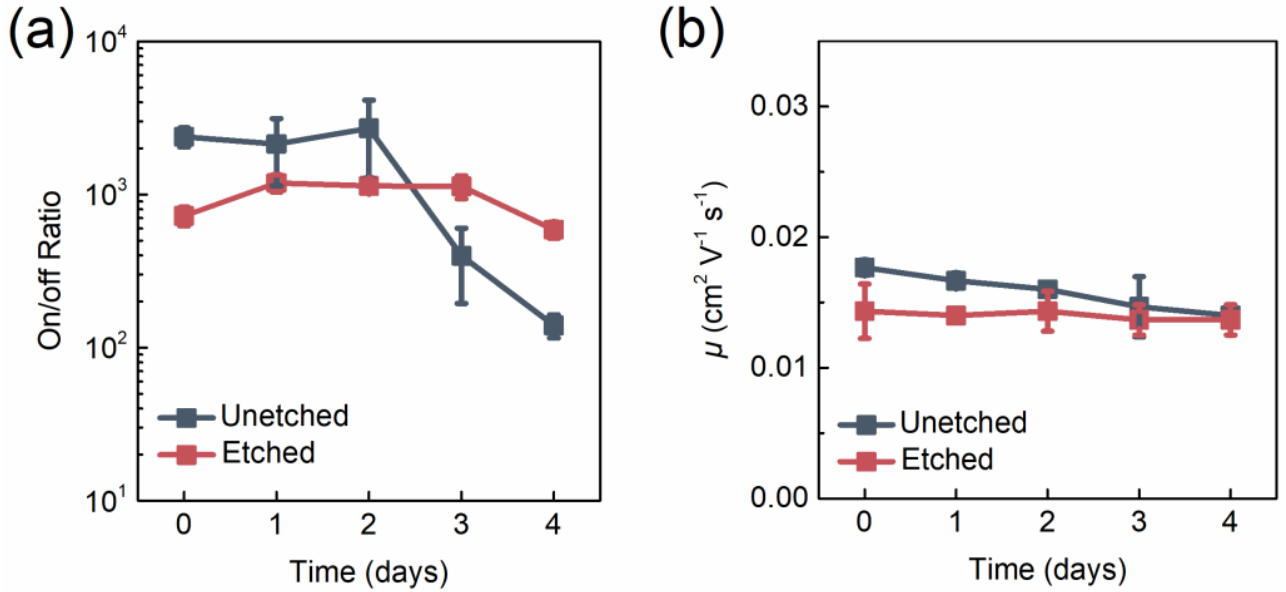

Figure S3. (a) On/off ratio and (b) field-effect mobility of unetched and etched P3HT transistors during exposure to air, which are extracted from transfer curves in Figures 2 and S1. 

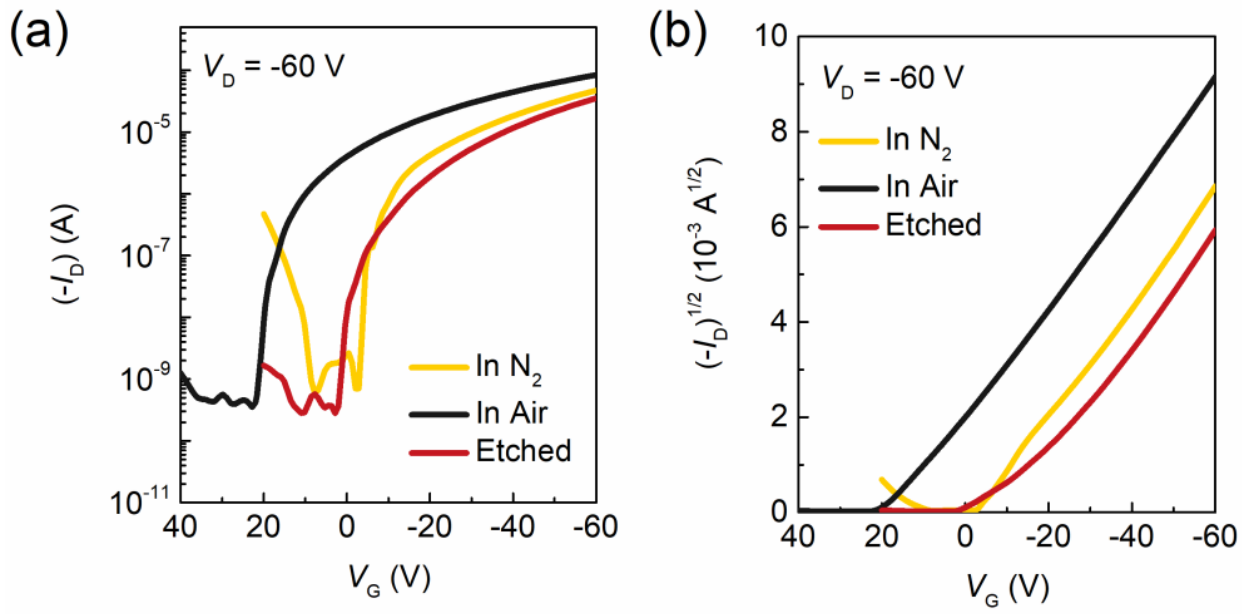

Figure S4. Transfer curves of PCDTPT transistors measured in different conditions. 
(a)

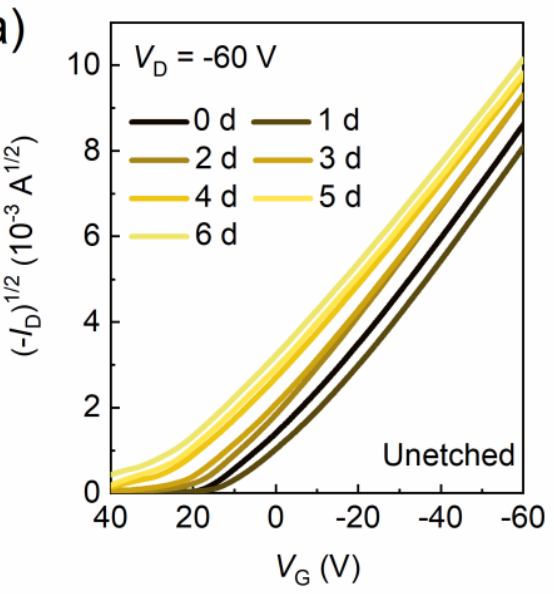

(b)

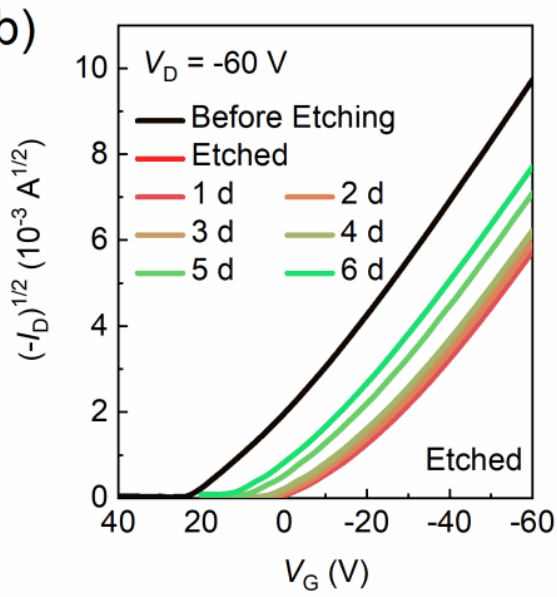

Figure S5. In situ evolution of transfer curves $\left(\left(-I_{D}\right)^{1 / 2}-V_{G}\right)$ for (a) unetched and (b) etched PCDTPT transistors during exposure to air. 

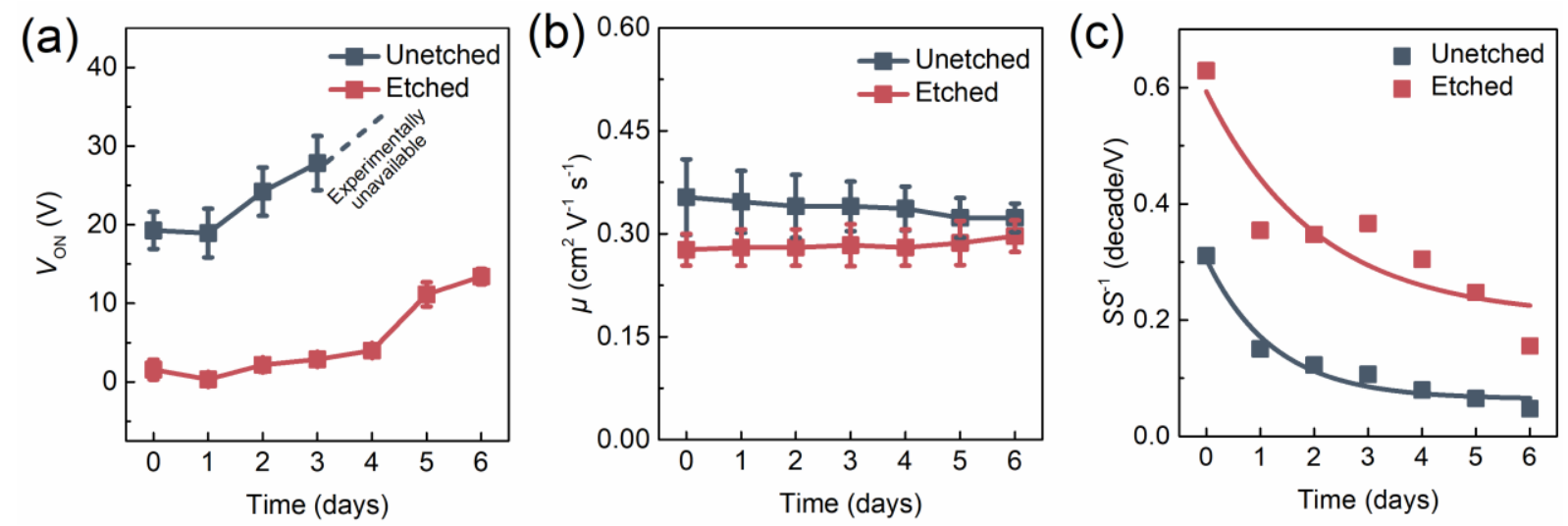

Figure S6. (a) $V_{\mathrm{ON}}$ and (b) field-effect mobility of unetched and etched PCDTPT transistors during exposure to air, which are extracted from transfer curves in Figure S5. In (a), for unetched PCDTPT transistor, well-defined $V_{\mathrm{ON}}$ was experimentally unavailable after 3 days as a result of the poor performance. (c) $S S^{-1}$ of unetched and etched PCDTPT transistors during exposure to air and an exponential-decay formula is used to numerically fit the decay of $S S^{-1}$. 
(a)

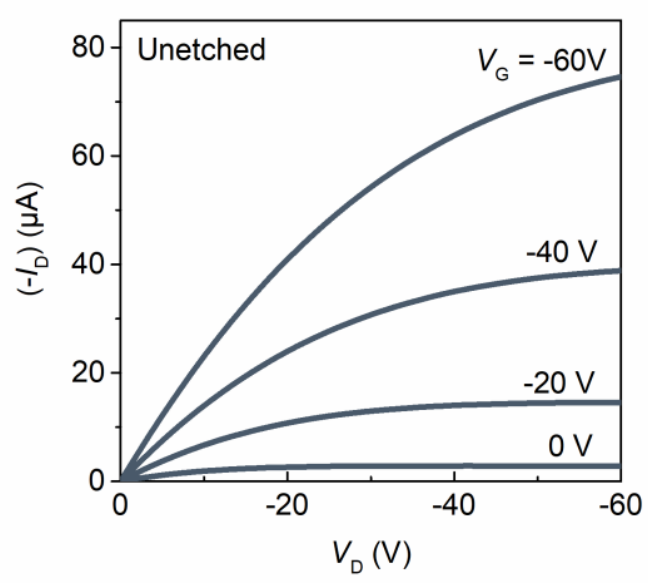

(b)

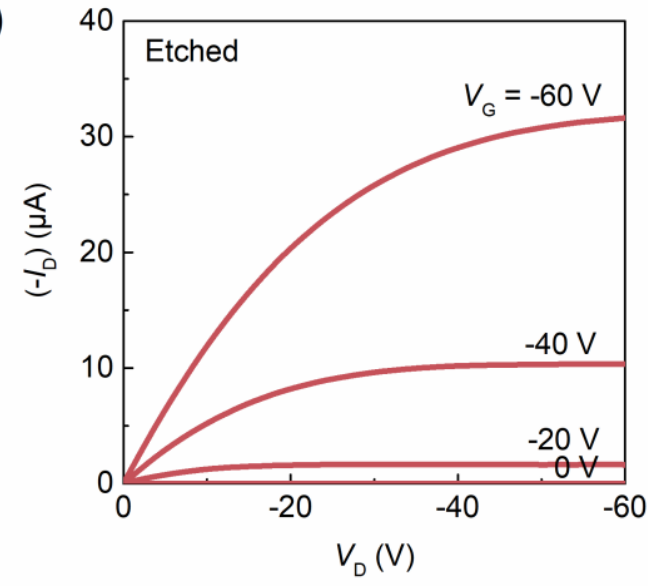

Figure S7. Output curves of (a) unetched and (b) etched PCDTPT transistors. The transfer curves are shown in Figure 3. 
(a)

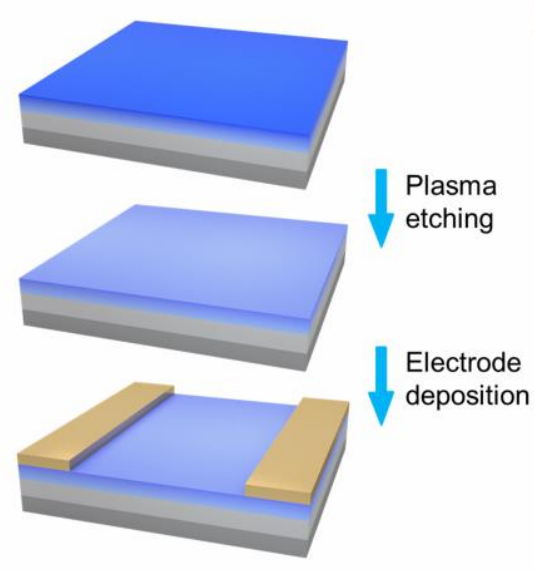

(b)

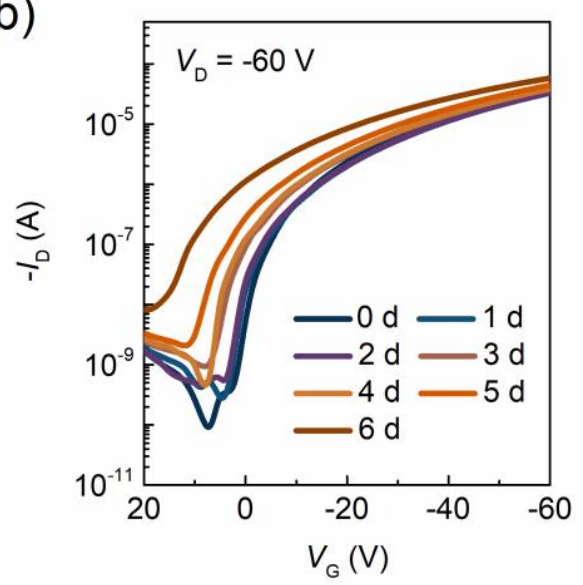

Figure S8. (a) The preparation process of OFETs that the etching of organic semiconductor film was performed before the deposition of source and drain electrodes. The resulting OFETs are different from the OFETs in Figure 3 that the etching of organic semiconductor film was performed after the deposition of source and drain electrodes. (b) In situ evolution of transfer curves during exposure to air for PCDTPT transistor based on (a). Compared with unetched PCDTPT device (Figure 3a), the stability of PCDTPT transistor based on (a) was improved. 
(a)
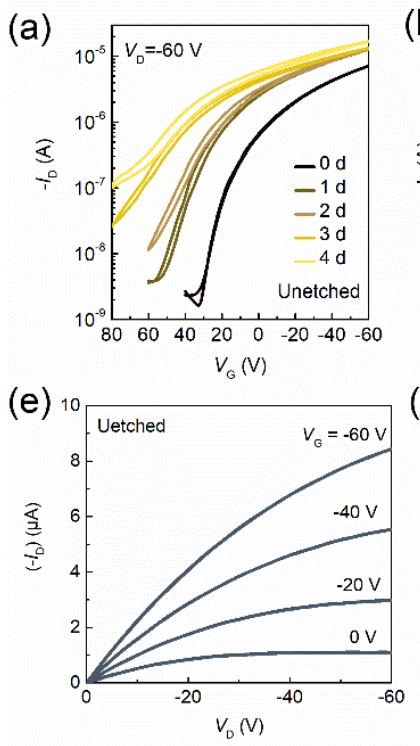

(b)

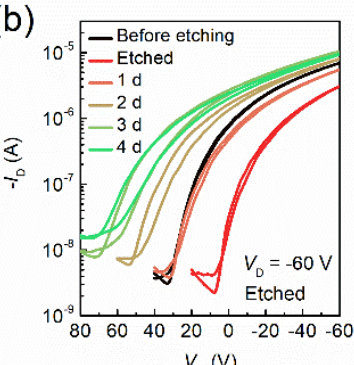

(f)

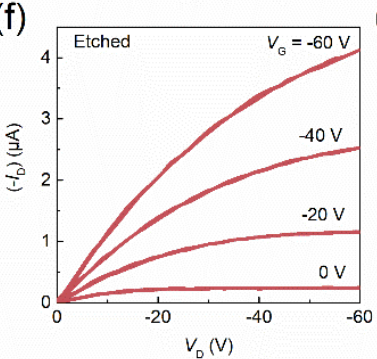

(c)

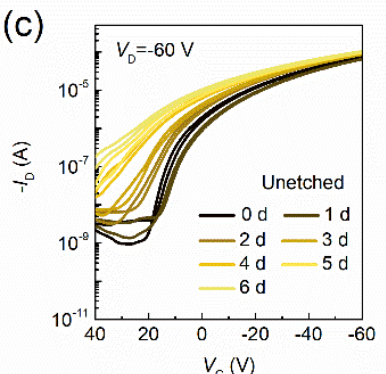

(g)

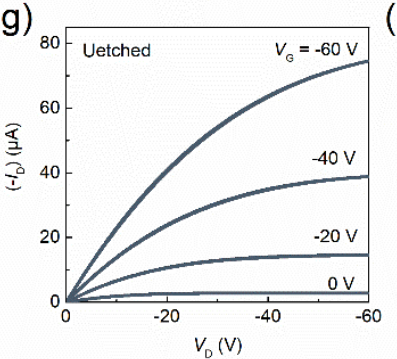

(d)

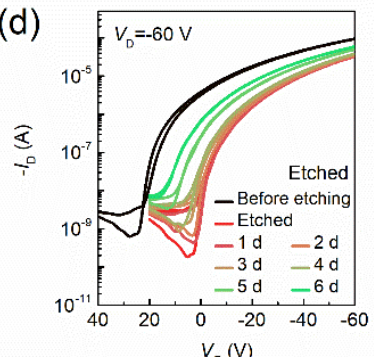

(h)

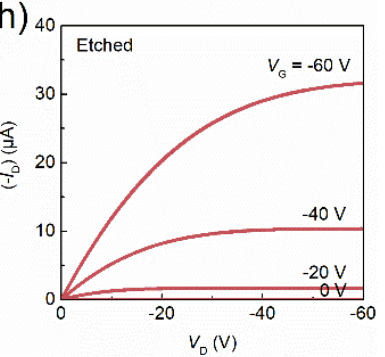

Figure S9. Dual sweep transfer curves of P3HT transistors ((a) unetched and (b) etched) and PCDTPT transistors ((c) unetched and (d) etched). Dual sweep output curves of P3HT transistors ((e) unetched and (f) etched) and PCDTPT transistors ((g) unetched and (h) etched). 

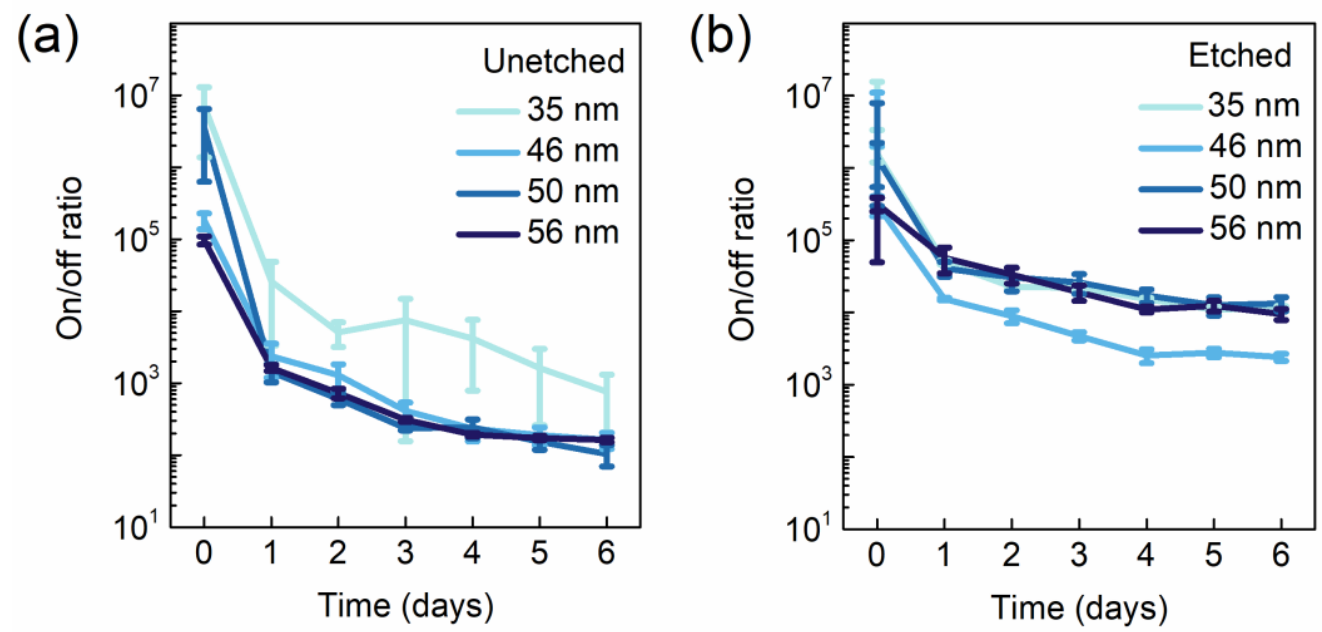

Figure S10. On/off ratio of (a) unetched and (b) etched PCDTPT transistors with different thicknesses during exposure to air. 


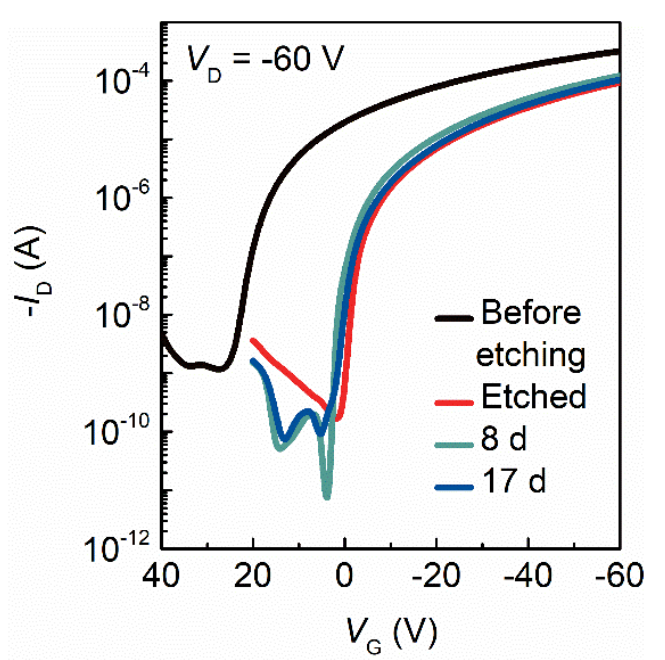

Figure S11. In situ transfer curves for etched PCDTPT transistor encapsulated and stored in $\mathrm{N}_{2}$. The PCDTPT transistor was encapsulated by CYTOP after surface etching. 
(a)
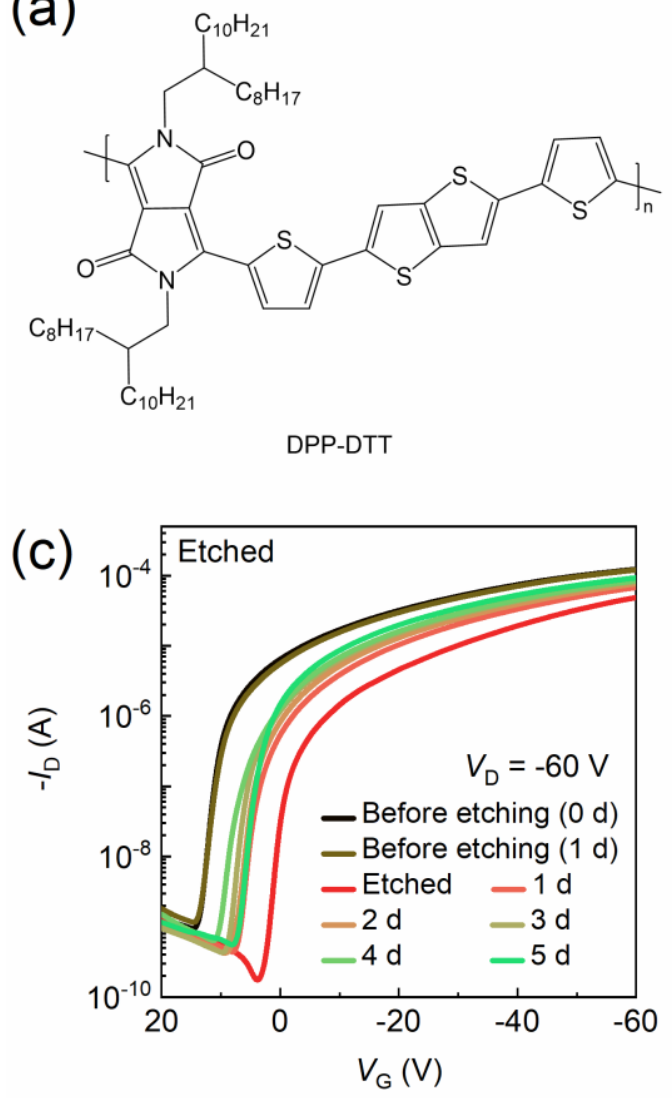

(b)

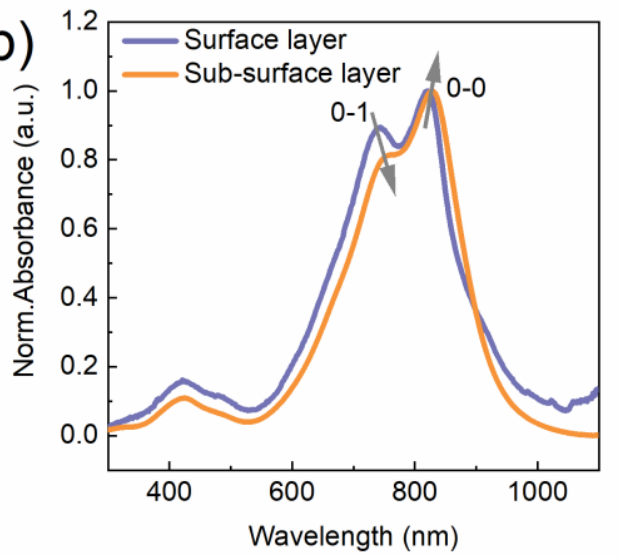

(d)

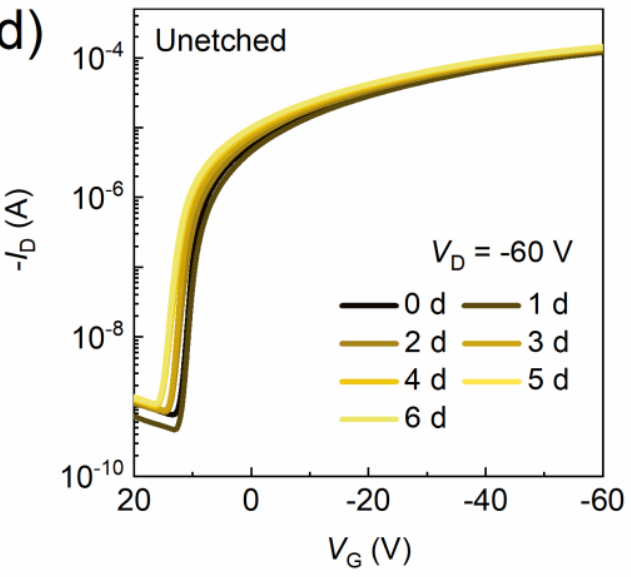

Figure S12. The effect of crystallinity on DPP-DTT transistors performance. (a) Chemical structure of DPP-DTT. (b) Normalized UV-vis absorption spectra of surface layer and subsurface layer in DPP-DTT film, which shows that the crystallinity of the surface layer is not higher than that of the subsurface layer. In situ evolution of transfer curves for (c) unetched and (d) etched DPP-DTT transistors during exposure to air, where the etching does not improve the device stability because in this case the surface does not have a higher crystallinity than the subsurface layer. 